\title{
On Separation Axioms and Sequences
}

\author{
ERDAL EKICI AND TAKASHI NOIRI
}

\begin{abstract}
In 2003, Noiri has introduced the notion of $\beta$ - $\theta$-open sets. By using these sets, the aim of this paper is to investigate the relationships between separation axioms and sequences.
\end{abstract}

\section{INTRODUCTION}

Noiri [10] has introduced the notion of $\beta-\theta$-open sets in topological spaces in 2003. Noiri has shown that $\beta-\theta$-open sets are weaker form of $\beta$-regular sets and stronger form of $\beta$-open sets. Separation axioms and sequences are two main topics of general topology. In literature, many papers have been studied on these subjects $[4,5,6,9,11,12$, etc.]. In this paper, we investigate the relationships between separation axioms and sequences by using $\beta$ - $\theta$-open sets.

\section{PReliminaries}

Throughout the present paper, spaces $X$ and $Y$ mean topological spaces. Let $X$ be a topological space and $A$ a subset of $X$. The closure of $A$ and the interior of $A$ are denoted by $\operatorname{cl}(A)$ and $\operatorname{int}(A)$, respectively.

A subset $A$ is said to be $\beta$-open [1] or semi-preopen [3] (resp. $\alpha$-open [8]) if $A \subset \operatorname{cl}(\operatorname{int}(\operatorname{cl}(A)))($ resp. $A \subset \operatorname{int}(\operatorname{cl}(\operatorname{int}(A))))$. The complement of a $\beta$-open set is said to be $\beta$-closed [2] or semi-preclosed [3]. The intersection of all $\beta$-closed sets of $X$ containing $A$ is called the semi-preclosure [3] or $\beta$-closure [2] of $A$ and is denoted by $\beta-\operatorname{cl}(A)$. The union of all $\beta$-open sets of $X$ contained in $A$ is called the semi-preinterior or $\beta$-interior of $A$ and is denoted by $\beta$-int $(A)$. A subset $A$ is said to be $\beta$-regular if it is $\beta$-open and $\beta$-closed.

The family of all $\beta$-open (resp. $\beta$-regular) sets of $X$ containing a point $x \in X$ is denoted by $\beta O(X, x)$ (resp. $\beta R(X, x)$ ). The family of all $\beta$-open (resp. $\beta$-closed, $\beta$-regular) sets in $X$ is denoted by $\beta O(X)$ (resp. $\beta C(X), \beta R(X)$ ).

A topological space $X$ is called $\beta-T_{0}$ [7] if for any distinct pair of points in $X$, there exists a $\beta$-open set containing one of the points but not the other. A space $X$ is said to be $\beta-T_{1}[7]$ if for each pair of distinct points $x$ and $y$ of $X$, there exist $\beta$-open sets $U$ and $V$ such that $x \in U, y \notin U$ and $x \notin V, y \in V$. A space $X$

2000 Mathematics Subject Classification. Primary: 54A05, 54D10.

Key words and phrases. separation axioms, sequences, $\beta$ - $\theta$-open sets. 
is said to be $\beta-T_{2}[7]$ if for each pair of distinct points $x$ and $y$ of $X$, there exist $\beta$-open sets $U$ and $V$ such that $x \in U, y \in V$ and $U \cap V=\emptyset$.

Theorem 2.1. ([10]) Let $A$ be a subset of a topological space $X$. Then

(1) $A \in \beta O(X)$ if and only if $\beta-\operatorname{cl}(A) \in \beta R(X)$,

(2) $A \in \beta C(X)$ if and only if $\beta$-int $(A) \in \beta R(X)$.

Definition 2.1. ([10]) Let $X$ be a topological space. A point $x$ of $X$ is called a $\beta-\theta$-cluster point of $S$ if $\beta$-cl $(U) \cap S \neq \varnothing$ for every $U \in \beta O(X, x)$. The set of all $\beta-\theta$-cluster points of $S$ is called the $\beta-\theta$-closure of $S$ and is denoted by $\beta-\theta$ $\operatorname{cl}(S)$. A subset $S$ is said to be $\beta-\theta$-closed if $S=\beta-\theta-c l(S)$. The complement of a $\beta-\theta$-closed set is said to be $\beta-\theta$-open.

Theorem 2.2. ([10]) For any subset $A$ of a topological space $X$, the following hold:

$$
\begin{aligned}
\beta-\theta-c l(A) & =\cap\{V: A \subset V \text { and } V \text { is } \beta \text { - } \theta \text {-closed }\} \\
& =\cap\{V: A \subset V \text { and } V \in \beta R(X)\} .
\end{aligned}
$$

Theorem 2.3. ([10]) Let $A$ and $B$ be any subsets of a topological space $X$. Then the following properties hold:

(1) $x \in \beta-\theta-c l(A)$ if and only if $V \cap A \neq \varnothing$ for each $V \in \beta R(X, x)$,

(2) if $A \subset B$, then $\beta-\theta-\operatorname{cl}(A) \subset \beta-\theta-\operatorname{cl}(B)$,

(3) $\beta-\theta-c l(\beta-\theta-c l(A))=\beta-\theta-c l(A)$,

(4) if $A_{\alpha}$ is $\beta$ - $\theta$-closed in $X$ for each $\alpha \in \Delta$, then $\underset{\alpha \in \Delta}{\cap} A_{\alpha}$ is $\beta$ - $\theta$-closed in $X$.

The family of all $\beta$ - $\theta$-open (resp. $\beta-\theta$-closed) sets of $X$ containing a point $x \in X$ is denoted by $\beta \theta O(X, x)$ (resp. $\beta \theta C(X, x)$ ). The family of all $\beta$ - $\theta$-open (resp. $\beta-\theta$-closed) sets in $X$ is denoted by $\beta \theta O(X)$ (resp. $\beta \theta C(X)$ ).

\section{Separation axioms and sequences}

In this section, we introduce and study $\beta$ - $\theta$-separation axioms, $\beta-\theta$-convergences and some functions. Also, we investegate the relationships among $\beta-\theta$-separation axioms, $\beta-\theta$-convergences and some functions.

Definition 3.1. A topological space $X$ is called $\beta-\theta-T_{0}$ if for any distinct pair of points in $X$, there exists a $\beta$ - $\theta$-open set containing one of the points but not the other.

Definition 3.2. A space $X$ is said to be $\beta-\theta-T_{1}$ if for each pair of distinct points $x$ and $y$ of $X$, there exist $\beta$ - $\theta$-open sets $U$ and $V$ such that $x \in U, y \notin U$ and $x \notin V, y \in V$.

Definition 3.3. A space $X$ is said to be $\beta-\theta-T_{2}$ if for each pair of distinct points $x$ and $y$ of $X$, there exist $\beta-\theta$-open sets $U$ and $V$ such that $x \in U, y \in V$ and $U \cap V=\emptyset$. 
Theorem 3.1. For a topological space $(X, \tau)$, the following properties are equivalent:

(1) $X$ is $\beta-\theta-T_{2}$,

(2) for each pair of distinct points $x$ and $y$ of $X$, there exist $\beta$-regular sets $U$ and $V$ such that $x \in U, y \in V$ and $U \cap V=\emptyset$,

(3) $X$ is $\beta-T_{2}$,

(4) for each pair of distinct points $x$ and $y$ of $X$, there exist $\beta$ - $\theta$-open and $\beta-\theta$-closed sets $U$ and $V$ such that $x \in U, y \in V$ and $U \cap V=\emptyset$.

Proof. $(1) \Leftrightarrow(2)$ It is obvious since $A$ is $\beta$ - $\theta$-open in $X$ if and only if for each $x \in A$ there exists $V \in \beta R(X, x)$ such that $x \in V \subset A$.

$(2) \Leftrightarrow(3)$ It is obvious that $A \in \beta O(X)$ if and only if $\beta-c l(A) \in \beta R(X)$ and $A \in \beta C(X)$ if and only if $\beta$-int $(A) \in \beta R(X)$.

$(2) \Leftrightarrow(4)$ It is obvious that $A \in \beta R(X)$ if and only if $A$ is $\beta$ - $\theta$-open and $\beta$ - $\theta$ closed.

Remark 3.1. Every $\beta-\theta-T_{0}$ space is $\beta-T_{0}$ and every $\beta-\theta-T_{1}$ space is $\beta-T_{1}$.

Example 3.1. Let $X=\{a, b, c\}$ and $\tau=\{\varnothing, X,\{a\}\}$. Then $(X, \tau)$ is $\beta-T_{0}$ but not $\beta-\theta-T_{0}$.

Question Does there exist a space which is $\beta-T_{1}$ and it is not $\beta-\theta-T_{1}$ ?

Definition 3.4. A topological space $X$ is said to be $\beta-\theta-R_{1}$ if for $x, y \in X$ with $\beta-\theta-c l(\{x\}) \neq \beta-\theta-c l(\{y\})$, there exist disjoint $\beta-\theta$-open sets $U$ and $V$ such that $\beta-\theta-c l(\{x\}) \subset U$ and $\beta-\theta-c l(\{y\}) \subset V$.

Theorem 3.2. A topological space $X$ is $\beta-\theta-T_{1}$ if and only if the singletons are $\beta-\theta$-closed sets.

Definition 3.5. Let $X$ be a topological space and $S \subset X$. The $\beta$ - $\theta$-kernel of $S$, denoted by $\beta-\theta-k e r(S)$, is defined to be the set $\beta-\theta-k e r(S)=\cap\{U \in \beta \theta O(X)$ : $S \subset U\}$.

Theorem 3.3. A topological space $X$ is $\beta-\theta-R_{1}$ if and only if there exist disjoint $\beta-\theta$-open sets $U$ and $V$ such that $\beta-\theta-c l(\{x\}) \subset U$ and $\beta-\theta-c l(\{y\}) \subset V$ whenever $\beta-\theta-\operatorname{ker}(\{x\}) \neq \beta-\theta-\operatorname{ker}(\{y\})$ for $x, y \in X$.

Theorem 3.4. A topological space $X$ is $\beta-\theta-T_{2}$ if and only if it is $\beta-\theta-R_{1}$ and $\beta-\theta-T_{0}$.

Proof. $(\Rightarrow)$ : Let $X$ be a $\beta-\theta-T_{2}$ space. Then $X$ is $\beta-\theta-T_{1}$ and then $\beta-\theta-T_{0}$. Since $X$ is $\beta-\theta-T_{2}$, by the Theorem 3.2, $\{x\}=\beta-\theta-c l(\{x\}) \neq \beta-\theta-c l(\{y\})=\{y\}$ for $x$, $y \in X$, there exist disjoint $\beta-\theta$-open sets $U$ and $V$ such that $\beta-\theta-c l(\{x\}) \subset U$ and $\beta-\theta-c l(\{y\}) \subset V$. Thus, $X$ is a $\beta-\theta-R_{1}$ space.

$(\Leftarrow)$ : Let $X$ be $\beta-\theta-R_{1}$ and $\beta-\theta-T_{0}$. Let $x, y$ be any two distinct points of $X$. Since $X$ is $\beta-\theta-\mathrm{T}_{0}$, then there exists a $\beta$ - $\theta$-open set $U$ such that $x \in U$ and $y \notin U$ or there exists a $\beta$ - $\theta$-open set $V$ such that $y \in V$ and $x \notin V$. Let $x \in U$ and $y \notin U$. Then $y \notin \beta-\theta-k e r(\{x\})$ and then $\beta$ - $\theta$-ker $(\{x\}) \neq \beta-\theta-k e r(\{y\})$. Since $X$ 
is $\beta-\theta-R_{1}$, by Theorem 3.3 there exist disjoint $\beta-\theta$-open sets $U$ and $V$ such that $x \in \beta-\theta-c l(\{x\}) \subset U$ and $y \in \beta-\theta-c l(\{y\}) \subset V$. Thus, $X$ is $\beta-\theta-T_{2}$.

Lemma 3.1. ([1]) Let $A$ and $Y$ be subsets of a space $X$. If $A \in \beta O(X)$ and $Y$ is $\alpha$-open in $X$, then $A \cap Y \in \beta O(Y)$.

Theorem 3.5. If $X_{0}$ be an $\alpha$-open set and $A$ be a $\beta-\theta$-open set in $X$, then $X_{0} \cap A \in \beta \theta O\left(X_{0}\right)$.

Definition 3.6. A sequence $\left(x_{n}\right)$ is said to be $\beta-\theta$-convergent to a point $x$ of $X$, denoted by $\left(x_{n}\right) \stackrel{\beta \theta}{\rightarrow} x$, if $\left(x_{n}\right)$ is eventually in every $\beta-\theta$-open set containing $x$.

Definition 3.7. A space $X$ is said to be $\beta-\theta-U S$ if every $\beta-\theta$-convergent sequence $\left(x_{n}\right)$ in $X \beta$ - $\theta$-converges to a unique point.

Definition 3.8. A set $F$ of a space $X$ is said to be sequentially $\beta$ - $\theta$-closed if every sequence in $F \beta$ - $\theta$-converging in $X \beta-\theta$-converges to a point in $F$.

Definition 3.9. A subset $G$ of a space $X$ is said to be sequentially $\beta$ - $\theta$-compact if every sequence in $G$ has a subsequence which $\beta-\theta$-converges to a point in $G$.

Theorem 3.6. Every $\beta-\theta-T_{2}$ space is $\beta-\theta-U S$.

Proof. Let $X$ be a $\beta-\theta-T_{2}$ space and $\left(x_{n}\right)$ be a sequence in $X$. Suppose that $\left(x_{n}\right)$ $\beta-\theta$-converges to two distinct points $x$ and $y$. That is, $\left(x_{n}\right)$ is eventually in every $\beta-\theta$-open set containing $x$ and also in every $\beta-\theta$-open set containing $y$. This is contradiction since $X$ is a $\beta-\theta-T_{2}$ space. Hence, the space $X$ is $\beta-\theta$-US.

Theorem 3.7. Every $\beta-\theta-U S$ space is $\beta-\theta-T_{1}$.

Proof. Let $X$ be a $\beta$ - $\theta$-US space. Let $x$ and $y$ be two distinct points of X. Consider the sequence $\left(x_{n}\right)$ where $x_{n}=x$ for every $n$. Clearly, $\left(x_{n}\right) \beta$ - $\theta$-converges to $x$. Also, since $x \neq y$ and $X$ is $\beta$ - $\theta$-US, $\left(x_{n}\right)$ cannot $\beta$ - $\theta$-converge to $y$, i.e, there exists a $\beta$ - $\theta$-open set $V$ containing $y$ but not $x$. Similarly, if we consider the sequence $\left(y_{n}\right)$ where $y_{n}=y$ for all $n$, and proceeding as above we get a $\beta$ - $\theta$-open set $U$ containing $x$ but not $y$. Thus, the space $X$ is $\beta-\theta-T_{1}$.

Theorem 3.8. A space $X$ is $\beta-\theta-U S$ if and only if the set $A=\{(x, x): x \in X\}$ is a sequentially $\beta$ - $\theta$-closed subset of $X \times X$.

Proof. Let $X$ be $\beta$ - $\theta$-US. Let $\left(x_{n}, x_{n}\right)$ be a sequence in $A$. Then $\left(x_{n}\right)$ is a sequence in $X$. As $X$ is $\beta$ - $\theta$-US, $\left(x_{n}\right) \stackrel{\beta \theta}{\rightarrow} x$ for a unique $x \in X$. i.e., $\left(x_{n}\right) \beta$ - $\theta$-converge to $x$ and $y$. Thus, $x=y$. Hence, $A$ is a sequentially $\beta$ - $\theta$-closed set.

Conversely, let $A$ be sequentially $\beta$ - $\theta$-closed. Let a sequence $\left(x_{n}\right) \beta$ - $\theta$-converge to $x$ and $y$. Hence, sequence $\left(x_{n}, x_{n}\right) \beta-\theta$-converges to $(x, y)$. Since $A$ is sequentially $\beta$ - $\theta$-closed, $(x, y) \in A$ which means that $x=y$ implies space $X$ is $\beta-\theta$-US.

Theorem 3.9. A space $X$ is $\beta-\theta-U S$ if and only if every sequentially $\beta$ - $\theta$-compact set of $X$ is sequentially $\beta-\theta$-closed. 
Proof. $(\Rightarrow)$ : Let $X$ be a $\beta$ - $\theta$-US space. Let $Y$ be a sequentially $\beta$ - $\theta$-compact subset of $X$. Let $\left(x_{n}\right)$ be a sequence in $Y$. Suppose that $\left(x_{n}\right) \beta$ - $\theta$-converges to a point $x$ in $X \backslash Y$. Let $\left(x_{n_{k}}\right)$ be a subsequence of $\left(x_{n}\right)$ which $\beta$ - $\theta$-converges to a point $y \in Y$ since $Y$ is sequentially $\beta$ - $\theta$-compact. Also, a subsequence $\left(x_{n_{k}}\right)$ of $\left(x_{n}\right) \beta$ - $\theta$-converges to $x \in X \backslash Y$. Since $\left(x_{n_{k}}\right)$ is a sequence in the $\beta$ - $\theta$-US space $X$, $x=y$. Thus, $Y$ is sequentially $\beta$ - $\theta$-closed set.

$(\Leftarrow)$ : Suppose that $\left(x_{n}\right)$ is a sequence in $X \beta$ - $\theta$-converging to distinct points $x$ and $y$. Let $K_{x}=\left\{x_{n}: n \in N\right\} \cup\{x\}$. Then $K_{x}$ is a sequentially $\beta$ - $\theta$-compact set and it is sequentially $\beta$ - $\theta$-closed. Since $y \notin K_{x}$, there exists $U \in \beta R(X, x)$ such that $U \cap K_{x}=\varnothing$. This contradicts that $\left(x_{n}\right)$ is eventually in $U$. Therefore, $x=y$ and $X$ is $\beta-\theta$-US.

Theorem 3.10. Every $\alpha$-open set of a $\beta-\theta-U S$ space is $\beta-\theta-U S$.

Proof. Let $X$ be a $\beta$ - $\theta$-US space and $Y \subset X$ be an $\alpha$-open set. Let $\left(x_{n}\right)$ be a sequence in $Y$. Suppose that $\left(x_{n}\right) \beta$ - $\theta$-converges to $x$ and $y$ in $Y$. We shall prove that $\left(x_{n}\right) \beta$ - $\theta$-converges to $x$ and $y$ in $X$. Let $U$ be any $\beta$ - $\theta$-open subset of $X$ containing $x$ and $V$ be any $\beta$ - $\theta$-open set of $X$ containing $y$. Then, $U \cap Y$ and $V \cap Y$ are $\beta$ - $\theta$-open sets in $Y$. Therefore, $\left(x_{n}\right)$ is eventually in $U \cap Y$ and $V \cap Y$ and so in $U$ and $V$. Since $X$ is $\beta$ - $\theta$-US, this implies that $x=y$. Hence the subspace $Y$ is $\beta-\theta-\mathrm{US}$.

Theorem 3.11. A space $X$ is $\beta-\theta-T_{2}$ if and only if it is both $\beta-\theta-R_{1}$ and $\beta-\theta-U S$.

Proof. Let $X$ be a $\beta-\theta-T_{2}$ space. Then $X$ is $\beta-\theta-R_{1}$ by Theorem 3.4 and $\beta-\theta$-US by Theorem 3.6.

Conversely, let $X$ be both $\beta-\theta-R_{1}$ and $\beta-\theta$-US space. By Theorem 3.7 we obtain that every $\beta-\theta$-US space is $\beta-\theta-T_{1}$ and $X$ is both $\beta-\theta-T_{1}$ and $\beta-\theta-R_{1}$ and, it follows from Theorem 3.4 that the space $X$ is $\beta-\theta-T_{2}$.

Next, we prove the product theorem for $\beta-\theta$-US spaces.

Theorem 3.12. If $X_{1}$ and $X_{2}$ are $\beta-\theta$-US spaces, then $X_{1} \times X_{2}$ is $\beta-\theta$-US.

Proof. Let $X=X_{1} \times X_{2}$ where $X_{i}$ is $\beta$ - $\theta$-US and $I=\{1,2\}$. Let a sequence $\left(x_{n}\right)$ in $X \beta$ - $\theta$-converge to $x=\left(x_{i}\right)_{i \in I}$ and $y=\left(y_{i}\right)_{i \in I}$. Then the sequence $\left(x_{n_{i}}\right)$ $\beta$ - $\theta$-converges to $x_{i}$ and $y_{i}$ for all $i \in I$. Suppose that for $2 \in I,\left(x_{n_{2}}\right)$ does not $\beta$ - $\theta$-converges to $x_{2}$. Then there exists $\beta$ - $\theta$-open set $U_{2}$ containing $x_{2}$ such that $\left(x_{n_{2}}\right)$ is not eventually in $U_{2}$. Consider the set, $U=X_{1} \times U_{2}$. Then $U$ is a $\beta-\theta$ open subset of $X$ and $x \in U$. Also, $\left(x_{n}\right)$ is not eventually in $U$ which contradicts the fact that $\left(x_{n}\right) \beta$ - $\theta$-converges to $x$. Thus we get $\left(x_{n_{i}}\right) \beta-\theta$-converges to $x_{i}$ and $y_{i}$ for all $i \in I$. Since $X_{i}$ is $\beta$ - $\theta$-US for each $i \in I$, we obtain $x=y$. Hence, $X$ is $\beta-\theta-\mathrm{US}$.

Now we define the notion of sequentially $\beta$ - $\theta$-continuous functions in the following:

Definition 3.10. A function $f: X \rightarrow Y$ is said to be 
(1) sequentially $\beta-\theta$-continuous at $x \in X$ if $f\left(x_{n}\right) \beta-\theta$-converges to $f(x)$ whenever $\left(x_{n}\right)$ is a sequence $\beta-\theta$-converging to $x$,

(2) sequentially $\beta$ - $\theta$-continuous if $f$ is sequentially $\beta-\theta$-continuous at all $x \in$ $X$.

Definition 3.11. A function $f: X \rightarrow Y$ is said to be sequentially nearly $\beta-\theta$ continuous if for each point $x \in X$ and each sequence $\left(x_{n}\right)$ in $X \beta$ - $\theta$-converging to $x$, there exists a subsequence $\left(x_{n_{k}}\right)$ of $\left(x_{n}\right)$ such that $f\left(x_{n_{k}}\right) \stackrel{\beta \theta}{\rightarrow} f(x)$.

Theorem 3.13. Let $f: X \rightarrow Y$ and $g: X \rightarrow Y$ be two sequentially $\beta-\theta$ continuous functions. If $Y$ is $\beta-\theta-U S$, then the set $A=\{x: f(x)=g(x)\}$ is sequentially $\beta$ - $\theta$-closed.

Proof. Let $Y$ be $\beta$ - $\theta$-US and suppose that there exists a sequence $\left(x_{n}\right)$ in $A \beta$ $\theta$-converging to $x \in X$. Since $f$ and $g$ are sequentially $\beta$ - $\theta$-continuous functions, $f\left(x_{n}\right) \stackrel{\beta \theta}{\longrightarrow} f(x)$ and $g\left(x_{n}\right) \stackrel{\beta \theta}{\rightarrow} g(x)$. Hence $f(x)=g(x)$ and $x \in A$. Therefore, $A$ is sequentially $\beta$ - $\theta$-closed.

Theorem 3.14. Let $f: X \rightarrow Y$ be a sequentially $\beta$ - $\theta$-continuous function. If $Y$ is $\beta-\theta-U S$, then the set $E=\{(x, y) \in X \times X: f(x)=f(y)\}$ is sequentially $\beta$ - $\theta$-closed in $X \times X$.

Proof. Suppose that there exists a sequence $\left(x_{n}, y_{n}\right)$ in $E \beta$ - $\theta$-converging to $(x, y) \in$ $X \times X$. Since $f$ is sequentially $\beta$ - $\theta$-continuous functions, $f\left(x_{n}\right) \stackrel{\beta \theta}{\rightarrow} f(x)$ and $f\left(y_{n}\right) \stackrel{\beta \theta}{\rightarrow} f(y)$. Hence $f(x)=f(y)$ and $(x, y) \in E$. Hence, $E$ is sequentially $\beta$ - $\theta$-closed.

Definition 3.12. A function $f: X \rightarrow Y$ is said to be sequentially sub- $\beta-\theta$ continuous if for each point $x \in X$ and each sequence $\left(x_{n}\right)$ in $X \beta$ - $\theta$-converging to $x$, there exists a subsequence $\left(x_{n_{k}}\right)$ of $\left(x_{n}\right)$ and a point $y \in Y$ such that $f\left(x_{n_{k}}\right) \stackrel{\beta \theta}{\longrightarrow}$ $y$.

Definition 3.13. A function $f: X \rightarrow Y$ is said to be sequentially $\beta$ - $\theta$-compact preserving if the image $f(K)$ of every sequentially $\beta$ - $\theta$-compact set $K$ of $X$ is sequentially $\beta$ - $\theta$-compact in $Y$.

Theorem 3.15. Every function $f: X \rightarrow Y$ is sequentially sub- $\beta$ - $\theta$-continuous if $Y$ is a sequentially $\beta$ - $\theta$-compact.

Proof. Let $\left(x_{n}\right)$ be a sequence in $X \beta$ - $\theta$-converging to a point $x$ of $X$. Then $\left(f\left(x_{n}\right)\right)$ is a sequence in $Y$ and as $Y$ is sequentially $\beta$ - $\theta$-compact, there exists a subsequence $\left(f\left(x_{n_{k}}\right)\right)$ of $\left(f\left(x_{n}\right)\right) \beta$ - $\theta$-converging to a point $y \in Y$. Hence, $f: X \rightarrow Y$ is sequentially sub- $\beta$ - $\theta$-continuous.

Theorem 3.16. A function $f: X \rightarrow Y$ is sequentially $\beta$ - $\theta$-compact preserving if and only if $f \mid M: M \rightarrow f(M)$ is sequentially sub- $\beta-\theta$-continuous for each sequentially $\beta$ - $\theta$-compact subset $M$ of $X$. 
Proof. Suppose that $f: X \rightarrow Y$ is a sequentially $\beta$ - $\theta$-compact preserving function. Then $f(M)$ is a sequentially $\beta$ - $\theta$-compact set in $Y$ for each sequentially $\beta-\theta$ compact set $M$ of $X$. Therefore, by Theorem 3.15, $f \mid M: M \rightarrow f(M)$ is a sequentially sub- $\beta-\theta$-continuous function.

Conversely, let $M$ be any sequentially $\beta$ - $\theta$-compact set of $X$. We shall show that $f(M)$ is a sequentially $\beta$ - $\theta$-compact set in $Y$. Let $\left(y_{n}\right)$ be any sequence in $f(M)$. Then for each positive integer $n$, there exists a point $x_{n} \in M$ such that $f\left(x_{n}\right)=y_{n}$. Since $\left(x_{n}\right)$ is a sequence in the sequentially $\beta$ - $\theta$-compact set $M$, there exists a subsequence $\left(x_{n_{k}}\right)$ of $\left(x_{n}\right) \beta$ - $\theta$-converging to a point $x \in M$. Since $f \mid M: M \rightarrow f(M)$ is sequentially sub- $\beta$ - $\theta$-continuous, then there exists a subsequence $\left(x_{n_{k}}\right)$ of $\left(x_{n}\right)$ such that $f\left(x_{n_{k}}\right) \stackrel{\beta \theta}{\rightarrow} y$ and $y \in f(M)$. This implies that $f(M)$ is a sequentially $\beta$ - $\theta$-compact set in $Y$. Thus, $f: X \rightarrow Y$ is a sequentially $\beta$ - $\theta$-compact preserving function.

The following theorem gives a sufficient condition for a sequentially sub- $\beta-\theta$ continuous function to be sequentially $\beta$ - $\theta$-compact preserving.

Theorem 3.17. If a function $f: X \rightarrow Y$ is sequentially sub- $\beta-\theta$-continuous and $f(M)$ is a sequentially $\beta$ - $\theta$-closed set in $Y$ for each sequentially $\beta$ - $\theta$-compact set $M$ of $X$, then $f$ is a sequentially $\beta$ - $\theta$-compact preserving function.

Proof. We use the previous theorem. It suffices to prove that $f \mid M: M \rightarrow f(M)$ is sequentially sub- $\beta-\theta$-continuous for each sequentially $\beta$ - $\theta$-compact subset $M$ of $X$. Let $\left(x_{n}\right)$ be any sequence in $M \beta-\theta$-converging to a point $x \in M$. Then since $f$ is sequentially sub- $\beta$ - $\theta$-continuous, there exist a subsequence $\left(x_{n_{k}}\right)$ of $\left(x_{n}\right)$ and a point $y \in Y$ such that $f\left(x_{n_{k}}\right) \beta$ - $\theta$-converges to $y$. Since $f\left(x_{n_{k}}\right)$ is a sequence in the sequentially $\beta$ - $\theta$-closed set $f(M)$ of $Y$, we obtain $y \in f(M)$. This implies that $f \mid M: M \rightarrow f(M)$ is sequentially sub- $\beta$ - $\theta$-continuous.

Theorem 3.18. Every sequentially nearly $\beta-\theta$-continuous function is sequentially $\beta$ - $\theta$-compact preserving.

Proof. Suppose that $f: X \rightarrow Y$ is a sequentially nearly $\beta$ - $\theta$-continuous function and let $M$ be any sequentially $\beta$ - $\theta$-compact subset of $X$. We shall show that $f(M)$ is a sequentially $\beta$ - $\theta$-compact set of $Y$. Let $\left(y_{n}\right)$ be any sequence in $f(M)$. Then for each positive integer $n$, there exists a point $x_{n} \in M$ such that $f\left(x_{n}\right)=y_{n}$. Since $\left(x_{n}\right)$ is a sequence in the sequentially $\beta$ - $\theta$-compact set $M$, there exists a subsequence $\left(x_{n_{k}}\right)$ of $\left(x_{n}\right) \beta$ - $\theta$-converging to a point $x \in M$. Since $f$ is sequentially nearly $\beta$ - $\theta$-continuous, then there exists a subsequence $\left(x_{j}\right)$ of $\left(x_{n_{k}}\right)$ such that $f\left(x_{j}\right) \stackrel{\beta \theta}{\rightarrow} f(x)$. Thus, there exists a subsequence $\left(y_{j}\right)$ of $\left(y_{n}\right) \beta$ - $\theta$-converging to $f(x) \in f(M)$. This shows that $f(M)$ is a sequentially $\beta$ - $\theta$-compact set in $Y$.

Theorem 3.19. Every sequentially $\beta-\theta$-compact preserving function is sequentially sub- $\beta-\theta$-continuous.

Proof. Suppose $f: X \rightarrow Y$ is a sequentially $\beta$ - $\theta$-compact preserving function. Let $x$ be any point of $X$ and $\left(x_{n}\right)$ any sequence in $X \beta$ - $\theta$-coverging to $x$. We 
shall denote the set $\left\{x_{n}: n=1,2,3, \ldots\right\}$ by $N$ and $M=N \cup\{x\}$. Then $M$ is sequentially $\beta$ - $\theta$-compact since $x_{n} \stackrel{\beta \theta}{\rightarrow} x$. Since $f$ is sequentially $\beta$ - $\theta$-compact preserving, it follows that $f(M)$ is a sequentially $\beta$ - $\theta$-compact set of $Y$. Since $\left(f\left(x_{n}\right)\right)$ is a sequence in $f(M)$, there exists a subsequence $\left(f\left(x_{n_{k}}\right)\right)$ of $\left(f\left(x_{n}\right)\right)$ $\beta$ - $\theta$-converging to a point $y \in f(M)$. This implies that $f$ is sequentially sub- $\beta-\theta$ continuous.

\section{REFERENCES}

[1] M.E. Abd El-Monsef, S.N. El-Deeb and R.A. Mahmoud, $\beta$-open sets and $\beta$-continuous mappings, Bull. Fac. Sci. Assiut Univ., 12 (1983), 77-90.

[2] M.E. Abd El-Monsef, R.A. Mahmoud and E.R. Lashin, $\beta$-closure and $\beta$-interior, J. Fac. Ed. Ain Shams Univ., 10 (1986), 235-245.

[3] D. Andrijević, Semi-preopen sets, Mat. Vesnik, 38 (1996), 24-32.

[4] C.E. Aull, Sequences in topological spaces, Comm. Math. (1968), 329-36.

[5] H.F. Cullen, Unique sequential limits, Boll. Un. Mat. Ital., 20 (1965), 123-124.

[6] A. Kar and P. Bhattacharyya, Some weak separation axioms, Bull. Calcutta Math. Soc., 82 (1990), 415-422.

[7] R.A. Mahmoud and M.E. El-Monsef, $\beta$-irresolute and $\beta$-topological invariant, Proc. Pakistan Acad. Sci., 27 (1990), 285-296.

[8] O. Njåstad, On some classes of nearly open sets, Pacific J. Math., 15 (1965), 961-970.

[9] T. Noiri, Sequentially subcontinuous functions, Atti Accad. Naz. Lincei Rend. Cl. Sci. Fis. Mat. Natur. (8), 58 (1975), 370-373.

[10] T. Noiri, Weak and strong forms of $\beta$-irresolute functions, Acta Math. Hungar., 99 (4) (2003), 315-328.

[11] T. Noiri and E. Hatir, $\wedge_{s p}$-sets and some weak separation axioms, Acta Math. Hungar., 103 (3) (2004), 225-232.

[12] A. Wilansky, Between $T_{1}$ and $T_{2}$, Amer. Math. Monthly, 74 (1967), 261-266.

\section{ERDAL EKICI}

DePARTMENT OF MATHEMATiCS

Canakkale Onsekiz Mart University

Terzioglu Campus

17020 CANAKKale

TURKEY

E-mail address: eekici@comu.edu.tr

TAKASHI NOIRI

2979-1 Shiokita-CHO, Hinagu

YATSUSHIRO-SHI, KUMAMOTO-KEN 869-5142

JAPAN

E-mail address: t.noiri@nifty.com 